\title{
Postoperative CEA and Other Non-traditional Risk Factors for Colon Cancer Recurrence: Findings from Swedish Population- Based Data
}

\author{
Mary R. Kwaan, MD, MPH ${ }^{1}$ \\ Division of General Surgery, Department of Surgery, David Geffen School of Medicine at UCLA, Los Angeles, CA
}

Adjuvant chemotherapy after curative resection of colon cancer is a critical intervention in our efforts to decrease cancer-specific mortality. Selection of the correct population for treatment is the subject of ongoing investigation and is particularly controversial in high-risk stage II patients. In this issue of Annals of Surgical Oncology, Osterman et al. ${ }^{1}$ use population-based data from Uppsala County in Sweden to investigate 'emerging' risk factors for colon cancer recurrence and mortality. 'Emerging' predictor variables [sidedness, pT3 and pT4 subclassification, lymph node (LN) ratio, tumor deposits, pre- and postoperative carcinoembryonic antigen (CEA) and C-reactive protein (CRP) levels] were distinguished from 'classic' risk factors (emergency surgery, $\mathrm{pT}$ and $\mathrm{pN}$ classification, low LN yield, malignancy grade, vascular and perineural invasion, and adjuvant treatment). Underpinning their study is the use of population-based data with granular detail, few missing data, and long duration (median 5.5 years) follow-up. Their cohort included 416 patients treated in Uppsala County in the modern era. In an adjusted analysis, after controlling for 'classic' risk factors, postoperative CEA $>5 \mathrm{ng} / \mathrm{mL}$, right-sided tumors, and nodal subclassification stages ( $\mathrm{pN} 1 \mathrm{a}, \mathrm{pN} 1 \mathrm{~b}$, etc.) were associated with recurrence.

Perhaps the most valuable finding from this study is that it confirms postoperative CEA, rather than preoperative $\mathrm{CEA}$, as a predictor of cancer recurrence. In a study of

\footnotetext{
(C) Society of Surgical Oncology 2019
}

First Received: 8 December 2019; Published Online: 18 December 2019

M. R. Kwaan, MD, MPH

e-mail: mkwaan@mednet.ucla.edu
1248 consecutive patients with resected stage I, II, and III cancer, elevated postoperative CEA $(>5 \mathrm{ng} / \mathrm{mL})$ was found to be associated with lower recurrence-free survival in stage III patients compared with patients with normal preoperative or normalized (elevated preoperatively, then normalized postoperatively) CEA, with the effect persisting in an adjusted analysis. ${ }^{2}$ In a recent analysis of MOSAIC (adjuvant FOLFOX vs. 5-fluorouracil colon cancer) and PETAAC-8 (adjuvant FOLFOX and cetuximab vs. FOLFOX alone in stage III cancer) randomized trial data with over 5 years of follow-up, postoperative CEA was independently predictive of disease-free and overall survival, after controlling for age, sex, treatment, $\mathrm{T}$ and $\mathrm{N}$ stage, BRAF mutation status, and mismatch repair status. Furthermore, substratification of CEA values into 0-1.3, $1.3-5$, and $>5 \mathrm{ng} / \mathrm{mL}$ added to the predictive power of both clinical trial cohorts. ${ }^{3}$

Due to the small sample size, the absence of correlation between recurrence and other risk factors found in the study by Osterman et al. should be considered less conclusive. Unexpectedly, some of the classic risk factors for recurrence, such as $\mathrm{T}$ stage, inadequate $\mathrm{LN}$ sampling, and vascular invasion, did not statistically correlate with recurrence. Additionally, tumor deposits, noted in $13 \%$ of patients, were not associated with recurrence or survival in the adjusted analysis, which could be attributed to a lack of statistical power in a small sample. Recent adjusted analyses of a larger sample, the National Cancer Database, found that tumor deposits were associated with worse survival in stage III patients, and further stratifies outcomes for LN-positive and LN-negative patients. ${ }^{4,5}$ Osterman et al. ${ }^{6}$ found that right-sided tumors, a variable of interest in patients with metastatic disease, was associated with recurrence, but their recent analysis using the larger national Swedish Colorectal Cancer registry did not find 
this to be a strong predictor of prognosis in curatively respected patients. Sidedness warrants further investigation; its predictive value may be confounded by more specific factors that were not available in this and other analyses, such as BRAF mutation or microsatellite instability.

In the Uppsala study, overall survival correlated with sidedness, LN ratio, elevated postoperative CEA levels, and elevated postoperative CRP in an adjusted analysis. Elevated postoperative CRP may point to the role that inflammation may have as a marker of tumor behavior, and it was correlated with multiple high-risk features such as $\mathrm{T}$ and $\mathrm{N}$ stage, poorly differentiated tumors, and vascular invasion; however, it was also associated with postoperative complications, which occurred in $28 \%$ of patients. Although the correlation between decreased survival and elevated postoperative CRP may be explained by the known adverse effect of major infectious surgical complications on long-term survival, ${ }^{7}$ authors did not tease this out in statistical models, weakening conclusions in regard to CRP and prognosis.

Further research on 'emerging' variables will be complemented by recent work on circulating tumor DNA (ctDNA), which can be measured in plasma. In a recent Australian study of 96 stage III patients, ctDNA was detected in $21 \%$ of patients after resection and $17 \%$ of patients after adjuvant chemotherapy. Detection of ctDNA predicted recurrence after surgery and after chemotherapy, with predictive power after adjustment for age, sex, $\mathrm{T}$ and $\mathrm{N}$ stage, and lymphovascular invasion (hazard ratio 7.5, 95\% confidence interval $3.5-16.1) .{ }^{8}$ While ctDNA is a promising bioassay that could serve to assess prognosis and also response to treatment, it would be expensive to incorporate into clinical practice and for now further work on other 'emerging' variables is still of value.

\section{REFERENCES}

1. Osterman E, Mezheyeuski A, Sjoblom T, Glimelius B. Beyond the NCCN risk factors in colon cancer: an evaluation in a Swedish population-based cohort. Ann Surg Oncol. https://doi.org/10.1245/ s10434-019-08148-3.

2. Konishi T, Shimada Y, Hsu M, et al. Association of preoperative ad postoperative serum carcinoembryonic antigen and colon cancer outcome. JAMA Oncol. 2018;4(3):309-15.

3. Auclin E, Taieb J, Lapage $\mathrm{C}$, et al. Carcinoembryonic antigen levels and survival in stage III colon cancer: post hoc analysis of the MOSAIC and PETACC-8 Trials. Cancer Epidemiol Biomark Prev. 2019;28:1153-61.

4. Mirkin KA, Kulaylat AS, Hollenbeak CS, Messaris E. Prognostic significance of tumor deposits in stage III colon cancer. Ann Surg Oncol. 2018;25:3179-84.

5. Wong-Chong N, Motl J, Hwang G, et al. Impact of tumor deposits on oncology outcomes in stage III colon cancer. Dis Colon Rectum. 2018;61:1043-52.

6. Osterman E, Glimelius B. Recurrence risk after up-to-date colon cancer staging, surgery, and pathology: analysis of the entire Swedish population. Dis Colon Rectum. 2018;61:1016-25.

7. Breugom AJ, van Dongen DT, Bastiaannet E, et al. Association between the most frequent complications after surgery for stage IIII colon cancer and short-term survival, long-term survival and recurrences. Ann Surg Oncol. 2016;23:2858-65.

8. Tie J, Cohen JD, Wang X, et al. Circulating tumor DNA analyses as markers of recurrence risk and benefit of adjuvant therapy for stage III colon cancer. JAMA Oncol. https://doi.org/10.1001/jama oncol.2019.3616.

Publisher's Note Springer Nature remains neutral with regard to jurisdictional claims in published maps and institutional affiliations. 